\title{
Analysis on the Application of the Artificial Intelligence Neural Network on the New Energy Micro Grid
}

\author{
Weiguo Yao
}

\author{
Xi'an International University, Xi'an Shaanxi, 710077
}

Keywords: Artificial Intelligence; Neural Network; Micro Grid of New Energy

\begin{abstract}
The electricity of the micro grid mainly comes from the wind power, but generating power by wind is not steady, it brings some negative influences to the whole electricity system. So, it is necessary to improve the forecasting accuracy of the generating capacity by wind. It can make the whole electric grid steady by the artificial intelligence. On the base of analyzing the neural network and the micro grid of the new energy, this thesis researches the specific application of the artificial intelligence neural network on the micro grid of the new energy, and puts forward the countermeasures for improving their approaches if they want to harness the artificial intelligence neural network well. The writer of this thesis hopes this thesis could provide some references for the development of the electric grid of China.
\end{abstract}

\section{Introduction}

Form the present developmental situation of the new energy in China, the proportion of the clean energy becomes larger and larger. Some kinds of the new energy develop quickly such as photovoltaic power, and wind power, and the new energy has been in the golden period of its development. The construction of the micro grid, the construction of the local grid, and the construction of the demonstrative area where the new energy electric power is occupying the higher proportions of the whole power supply are becoming the key points of the development of new energy micro grid. We think that to develop the micro grid of the new energy would become the developmental trend of the energy in future ${ }^{[1]}$; it is the effective supplement of the main grid, and it conforms to the direction of the reform on the energy structure and the electric power system in China. Especially, the advantages of the artificial intelligence neural network are becoming clear increasingly.

\section{Artificial intelligence neural network}

Artificial intelligence, which is abbreviated AI for short, is a rising technology science that researches on the theory, method, technique, and the application for simulating and expanding the intelligence of humankind ${ }^{[2]}$. As the constant development of the artificial intelligence, the relevant theory and technology about the artificial intelligence neural network are becoming more advanced, and they have been applied widely and deeply in many fields. 


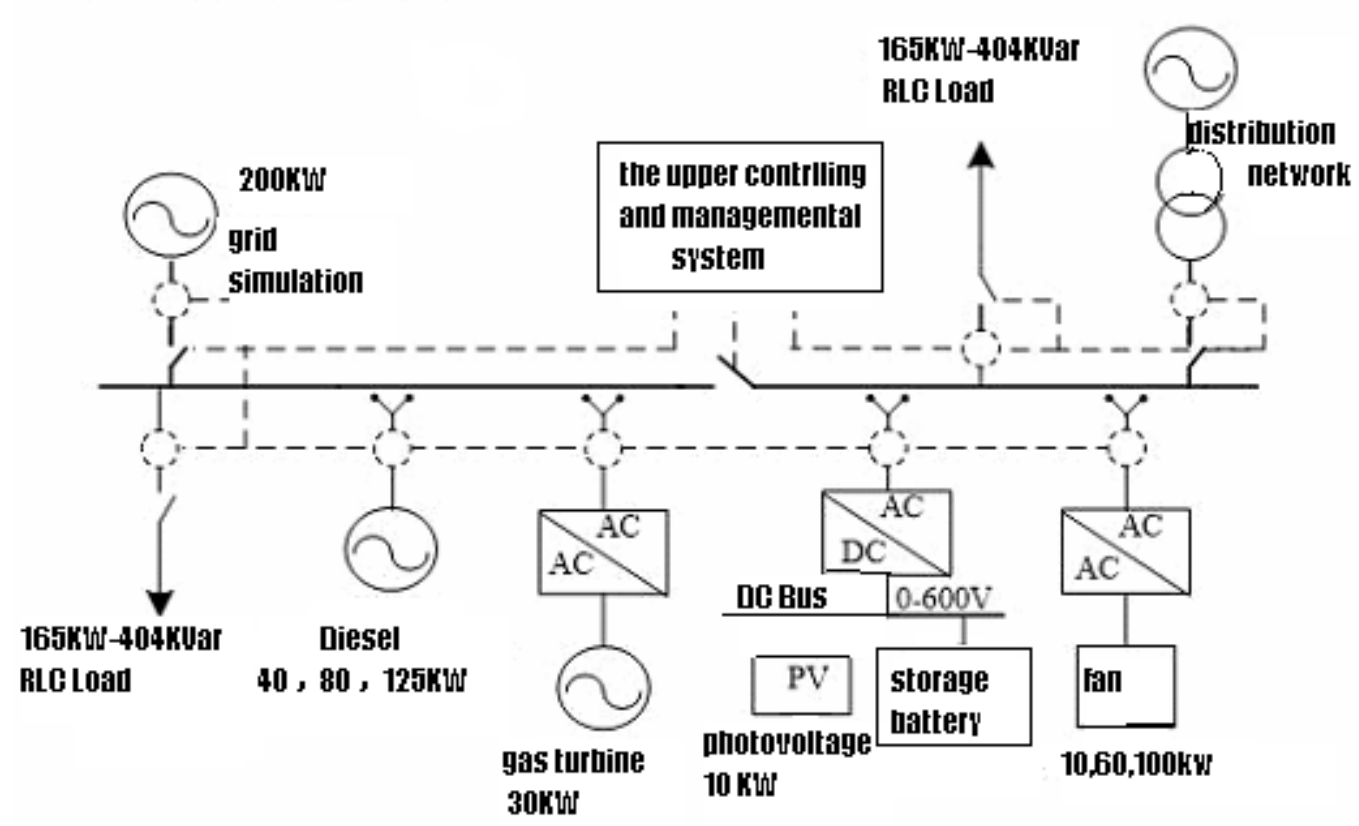

Chart1. The simple framework of the micro grid

To apply the artificial intelligence neural network is very significant to the electric enterprises on improving their productivities, the qualities of their productions, safety of the productions, and the stabilities of the electricity. For example, the Google Date Center utilizes the robot that is named DeepMind, which has learned some artificial intelligence, can effectively save $40 \%$ energy consumption. According to the analysis and forecast by the McKinsey Global Institute in their researching report----A Future That Works: Automation, Employment, and Productivity, it is estimated that automation could raise productivity growth globally by 0.8 to 1.4 percent annually.

\section{The micro grid of the new energy}

For the electricity enterprises, the micro electric grid provides the obvious conveniences on connecting the new energy electricity into the main network; it especially shows the obvious advantages on providing the electricity to the remote areas ${ }^{[4]}$. China has paid more attention to the development of the new energy micro grid gradually. In 2015, the National Energy Administration of China publishes----Guiding Opinions on Promoting the Construction of the Demonstrative Projects of New Energy Micro Grid----this document means to develop the micro electric grid has become a national strategy.

The new energy electricity comes from wind, solar, etc; although these kinds of energy are reproducible, but these energies are so unsteady that there are a lot of problems such as the electric generating efficiency is low, it is hard to forecast, and it is hard to manage, etc. After the deeply analysis we can find out that it is difficult to set up a model by the conventional technologies due to the complex and nonlinear relationship between the electric load and the other influencing factors. Especially, there are great differences between the main grid and the micro grid; it is also difficult to forecast the short-term electric load because the micro electric grid is more unsteady and more fluctuant, but we could forecast the generating capacity by wind well by means of the artificial intelligence neural network because the network has some advantages such as its flexible function model. For example, the EcoStruxure platform of the Schneider Electric can provide the large-scale solution to the user, such as managing the medium and low voltage circuit breaker at the worksite or via the network. As the increase of the collected data, we could improve the operation efficiency 
further more because of the modified algorithm ${ }^{[4]}$, and provide the better solution to the user in the terminal equipment. This thesis focuses on the micro grid of the new energy and the artificial intelligence neural network, and then analyzes the generating capacity by wind and the short-term forecasting methods of the new energy micro grid.

Analysis on the specific application of the artificial intelligence neural network on the micro grid of the new energy.

The RBF (Radial Basis Function) neural network is composed of the input layer, the hidden layer, and the output layer; the forecast model of the RBF neural network is shown as chart2 below. The input layer is composed of the nodes of the signal source; while the number of the second layer's nodes is determined by the issue that is discussed; the third layer responds to the function of the input model. The transform that are from the first layer to the second layer is a nonlinear function. By utilizing the artificial intelligence neural network, we could take the RBF as the "basis" of the hidden layer, so as to construct a complete hidden space. By input the different variable quantities, we can transform the original low dimension model into the high dimension model; at the some time, we also can ensure the convergence rate by artificial intelligence neural network. At present, the network is well utilized in many fields such as nonlinear function approximation, information processing, and system modeling, etc.

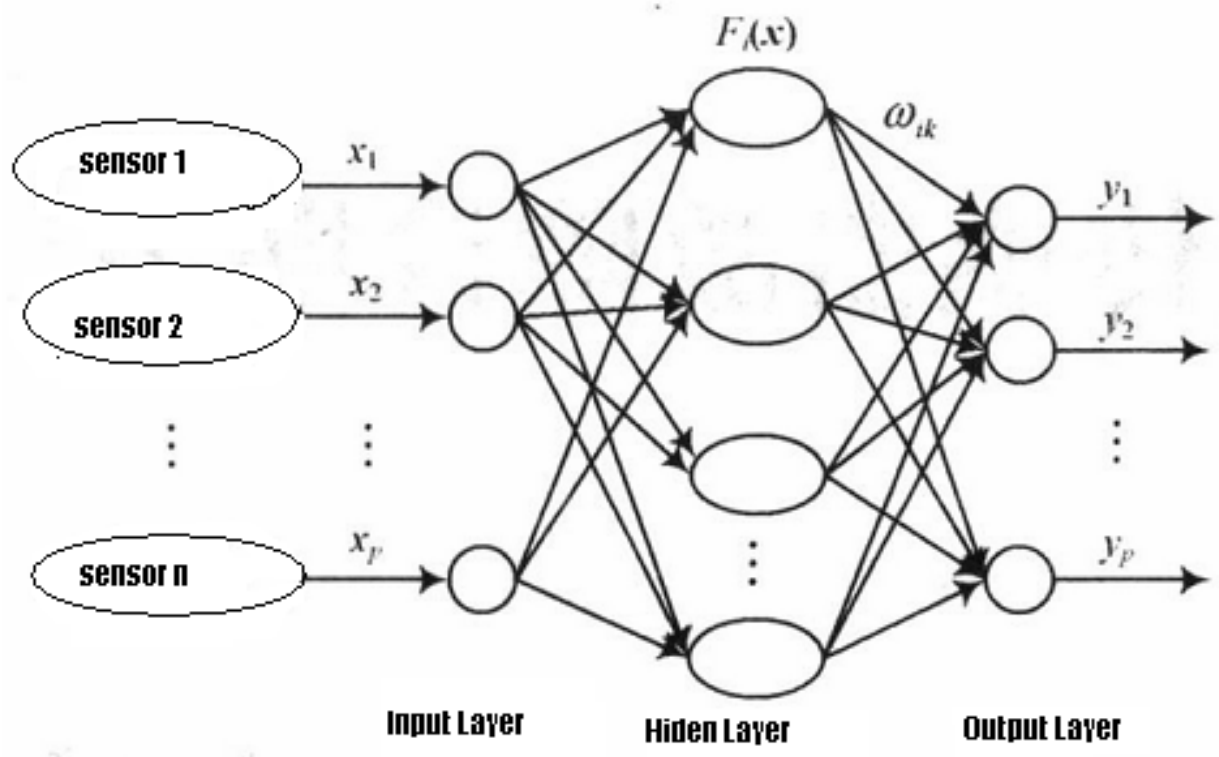

Chart 2.the forecast model of the neural network

By forecasting the short-term electric load, we can get to some help on the energy management of the new energy micro grid, and then promote the whole grid works economically, steadily, and smoothly. If we want to forecast and analysis on the short-term electric load of the micro grid well, we must collect the input data first; we should collect the relevant data that are highly related to the load, such as date, month, time, temperature, and wind speed, the load before a week, the maximum and the minimum load before a week, etc. We should choose the present load as the targeted load, and the forecasting result of the short-term load would be the output data ${ }^{[5]}$. After we confirm all the relevant data, we should unitize these data so as to get to the result. By the network training, we could establish a short-term forecast of the electric load that is on the base of the RBF neural network. We could refer to the sheet1 for the forecasting day and the numbers of the network training as below. 


\begin{tabular}{|c|c|c|c|c|}
\hline Date & \multicolumn{3}{|c|}{ Forecasting Day } & \multirow{2}{*}{$\begin{array}{c}\text { Numbers of } \\
\text { the Network } \\
\text { Training } \\
803 \\
\end{array}$} \\
\hline Monday & 1st, May & 21st, Aug & 20th, Dec & \\
\hline Tuesday & 2nd, May & 22nd, Aug & 21st, Dec & 854 \\
\hline Wednesday & 3rd, May & 23rd, Aug & 22nd, Dec & 878 \\
\hline Thursday & 4th, May & 24th, Aug & 23rd, Dec & 898 \\
\hline Friday & 5th, May & 25th, Aug & 24th, Dec & 924 \\
\hline Saturday & 6th, May & 26th, Aug & 25th, Dec & 956 \\
\hline Sunday & 7th, may & 27th, Aug & 26th, Dec & 1056 \\
\hline
\end{tabular}

Sheet1.The forecasting day and the numbers

Of the network training in different weeks

Considering the influence of the different season in the research, we choose May, August, and December these three months for discussing. We input the relevant specimens to the short-term forecast model of the RBF neural network, and then process the MATLAB simulative calculation. By the following analysis we find out that the average value of the MAPE of the short-term load forecast in every week is lower that $10 \%$, so the result can prove that the short-term forecast of electric load are feasible to the micro grid of new energy.

The advantages of the artificial intelligence neural network are obvious in the specific application. For example, the DeepMind that is subordinate to Google Inc. has constructed the cloud platform according to the artificial intelligence, the cloud platform could forecast the changes of the electric load by the neural network recognition system; it has saved $40 \%$ energy for the DeepMind by the computer severs and the relevant cooling systems such as the water pump, cooling tower, water chilling units, dry coolers etc; it also increasing the overall energy efficiency of the Google Inc. 15\%. In the new times, the electricity enterprises should adopt a lot of countermeasures so as to keep a faster developmental speed and become stronger and stronger.

It is very important to the management of the new energy micro grid by means of forecasting the wind speed and the generating capacity by wind according to the artificial intelligence neural network. Because the randomness and the volatility of the generating by wind ${ }^{[6]}$, it could easily influence the stability of the micro grid, so we should pay more attention to the randomness and the volatility of the generating process, so as to control the whole micro grid well.

Considering the wind speed is fluctuating in different seasons, if we take the wind speed and the atmospheric pressure as the input data of the input lay, the time would be a factor that we must think about a lot. By analyzing the relevant data of generating capacity of a community and the forecasting result of the wind speed, we could get to the approximate generating capacity of the 24 hours in the future; it is beneficial on maximizing the use of wind for generating.

The specific countermeasures of the electricity enterprises on utilizing the artificial intelligence neural network.

Because of the great developmental prospects of the new energy, and because of the current managerial problems of the electric industry, the electricity enterprises should improve the whole electric system by the artificial intelligence neural network. To generate electricity by the new energy can not only optimize the energy structure of the electricity industry, but also promote the construction of the environment friendly and resource conserving society. From the developmental perspective of our country, it is very significant to the development of China. Especially, China has achieves a lot of achievements on the development of the smart grid. For example, the Yibin Branch 
of the National Grid will invest approximate 2.293 billion on the construction of the electric grid that is lower than $500 \mathrm{KV}$, so as to construct a stronger smart local grid and promote the development of the local economy and society.

China has not only highlighted the construction of the smart grid, but also invested a lot of money on it. During the developmental process of the smart grid, it is very important to timely get to the generating capacity by wind and the forecast of the need of the users. However, if we want to ensure the artificial intelligence neural network could play a more important role in the new energy micro grid, it is necessary to strengthen the construction of the relevant infrastructures.

(1) To improve the intelligent level of the grid. We should try out best to construct a smart grid by all kinds of methods, such as utilizing the advantages of the mobile terminal equipments; optimizing the overall arrangement of the components on sensing, information, communicating, and controlling, so as to effectively strengthen the abilities of the grid on real-time getting to the relevant information and feeding back.

(2) The electric enterprises should utilize the opportunity of constructing the smart grid to connect the smart grid with the other relevant information systems such as heating pipeline, natural gas pipeline, and the traffic network ${ }^{[7]}$. The electricity enterprises should harness their advantages that could construct the grid freely, flexibly, so as to meet the plug-and-play demands of the smart external equipment, and to meet the demands of the distributed electric source and micro grid. Let the network becomes the foundation of all kinds of business in the future.

\section{Conclusion}

By the integration of the artificial intelligence neural network and the new energy micro grid, and by the forecast of the short-term electric load and the generating capacity, we can find out that the tested results are steady, and it has the great guiding significance on the management of the electric grid and the development of the smart grid; it is also significant to the improvement of the whole micro grid of the new energy. This thesis not only researches the artificial intelligence neural network, but also researches the present situation of new energy electric gird of China, so as to summarize the achievements of the electricity industry in China; the writer of this thesis hopes this thesis could provide some references to the electricity enterprises on the development of the reproducible energy and the clean energy.

\section{References}

[1]Li Jian. Research on the Application of the Artificial Intelligence on the Distinction of the Feature of Rocks and the Forecast of the Porosity and the Permeability----Take the Shihongtan Uranium Mine For Example[J]. Northwestern Geology, 2010,43(02):32-37.

[2]Chen Bingmei, Fan Xiaoping, Zhou Zhiming, Li Xuerong. Research on the Application of the artificial neural network and the artificial intelligence computer on the Diagnosis of psychological disorders of children[J]. Journal of Clinical Rehabilitative Tissue Engineering Research, 2011, 15(13):2467-2470.

[3]Liu Jingwei, Zhao Hui, Zhou Rui, Wang Pu. Exploration of High-Precision Adaptive Wavelet Neural Network Artificial Intelligence Method[J]. Journal of Frontiers of Computer Science and Technology, 2016, 10(08):1122-1132.

[4]Su Youli, Tang Jianguo. Application of Artificial Intelligence Neural Network in Micro Grid[J]. Journal of Xinjiang University(Science \& Engineering), 2016,33(04):444-448. 
[5]Liu Chunxia, Zhang Xueyan. Research on How to Improving the Forecast of the Short-term Electric Load That is Based on the Artificial Intelligence Neural Network[J]. Electro Technical Application, 2013, 32(04):74-77.

[6]Wan Yitai. Research on the Topology Structure and the Algorithm of the BP Artificial Intelligence Neural Network[J]. Heilongjiang Science and Technology Information, 2009, (28):84-85.

[7]Chen Yunji. From the Artificial Intelligence to the Processor of the Neural Network[J], The Forum of Leadership Science, 2017,(02):38-55. 